\title{
Reliability and Validity of Isometric Knee Extensor Strength Test With Hand-Held Dynamometer Depending on Its Fixation: A Pilot Study
}

\author{
Won Kuel Kim, MD, Don-Kyu Kim, MD, Kyung Mook Seo, MD, Si Hyun Kang, MD
}

Department of Physical Medicine and Rehabilitation, Chung-Ang University College of Medicine, Seoul, Korea

Objective To determine the reliability and validity of hand-held dynamometer (HHD) depending on its fixation in measuring isometric knee extensor strength by comparing the results with an isokinetic dynamometer.

Methods Twenty-seven healthy female volunteers participated in this study. The subjects were tested in seated and supine position using three measurement methods: isometric knee extension by isokinetic dynamometer, non-fixed HHD, and fixed HHD. During the measurement, the knee joints of subjects were fixed at a $35^{\circ}$ angle from the extended position. The fixed HHD measurement was conducted with the HHD fixed to distal tibia with a Velcro strap; non-fixed HHD was performed with a hand-held method without Velcro fixation. All the measurements were repeated three times and among them, the maximum values of peak torque were used for the analysis.

Results The data from the fixed HHD method showed higher validity than the non-fixed method compared with the results of the isokinetic dynamometer. Pearson correlation coefficients (r) between fixed HHD and isokinetic dynamometer method were statistically significant (supine-right: $r=0.806, p<0.05$; seating-right: $r=0.473, p<0.05$; supine-left: $\mathrm{r}=0.524, \mathrm{p}<0.05)$, whereas Pearson correlation coefficients between non-fixed dynamometer and isokinetic dynamometer methods were not statistically significant, except for the result of the supine position of the left leg $(\mathrm{r}=0.384, \mathrm{p}<0.05)$. Both fixed and non-fixed HHD methods showed excellent inter-rater reliability. However, the fixed HHD method showed a higher reliability than the non-fixed HHD method by considering the intraclass correlation coefficient (fixed HHD, 0.952-0.984; non-fixed HHD, 0.940-0.963).

Conclusion Fixation of HHD during measurement in the supine position increases the reliability and validity in measuring the quadriceps strength.

Keywords Muscle strength dynamometer, Isometric contraction, Physical restraint, Dimensional measurement accuracy, Knee joint 


\section{INTRODUCTION}

Assessment of muscle strength is one of the important predictive factors not only of neurological diseases or musculoskeletal disorders, but also of the physical function of elderly people. In particular, the lower extremity muscle strength is known as an important indicator of gait and physical functions of the elderly population $[1,2]$. Most recently, handgrip strength has been known to be highly associated with the lower extremity muscle power, the knee extensor torque and the cross-sectional area of the calf muscles among the elderly [3,4], and accordingly, has been used for clinical or research purposes in the geriatric field. However, this method is not for a direct measurement of the lower extremity muscle strength and many confusing factors affect the reliability and validity of the handgrip strength method. Moreover, the absence of a standard measurement method has limited its clinical applications $[5,6]$.

Isokinetic dynamometer has been used as a reliable and objective equipment for measuring the lower extremity muscle strength [7-10]. However, it is expensive, requires separate space, is not portable, requires a long measurement time and a trained rater, and may impose a heavy cost burden on researchers or patients. Accordingly, this method has been used for research purposes, but not often for large-scale studies.

In addition to the isokinetic dynamometer, the fixed dynamometer and hand-held dynamometer (HHD), which are portable devices, have been used for muscle strength measurement [11]. The fixed dynamometer has been used for research in a few studies, and has been primarily used for the fixation of dynamometer in order to avoid rater interference. However, it cannot be used for portable purposes because it needs to be fixed on to a column or wall in order to anchor it; further, it requires additional equipment and therefore is seldom used for research or clinical purposes at present. In addition, the direction of muscle strength measurement and dynamometer should be placed on the same line as the anchor; thus, there are limitations in its measurement position, joint angle and measurement site. Accordingly, its reliability is inferior to that of HDD in measuring the strength of some muscles $[12,13]$. In comparison, HHD can be directly attached to a specific body area in any position; however, it also has limitations in its measurement position, joint angle, measurement site, measurement type, muscle contraction type and speed. These limitations are also seen in the fixed dynamometer. Moreover, it has rater factor problems, such as the muscle strength and proficiency of the raters, which can affect the test results [14]. According to the systematic literature review conducted by Stark et al. [15], no standard HHD method that can verify the appropriate levels of reliability and validity has been established yet; hence, it cannot completely replace the isokinetic dynamometer test in clinical cases. Nevertheless, Thorborg et al. [16] reported that the HDD test fixating the device for measuring the muscle strength near the hip joint had reduced the rater errors that were observed with HHD and also had improved its reliability.

When the reliability of HDD, which is easy to carry and use, is enhanced and its standard protocol is available, it can be a highly recommendable method of muscle strength assessment. In this study, the peak knee extensor torque was measured using the typical non-fixed HHD method of holding the HDD and the fixed method; then, the results were compared with those of the isokinetic dynamometer method in order 1) to confirm their validity, 2) to assess their reliability through a test-retest method, and 3) to confirm the difference in the knee extensor muscle strength results of the HDD test, according to the measurement position. Based on the outcomes, the ideal measurement method and position are suggested in this study by confirming the reliability and validity of the HHD test, which is for measuring the knee extensor muscle strength.

\section{MATERIALS AND METHODS}

The study protocol was approved from the Institutional Review Board of Chung-Ang University Hospital. The subjects were recruited through advertisements and were informed of the purpose and nature of this study before they signed the written consent form.

\section{Subject}

Of the 30 recruited women in their 20 s and 30 s, three who were suffering from musculoskeletal diseases at the time of their recruitment; who had undergone musculoskeletal surgery that could have affected both their legs; who had abnormal physical examination findings in their knee or hip joint; who had a history of diabetes, 
thyroidism or hypertension; or who performed vigorous exercises that could affect their muscle strength during the study period were excluded from this study. Eventually, 27 healthy young women were eligible in this study. The mean age of the subjects was $23.9 \pm 2.9$ years; their mean height, $162.0 \pm 5.1 \mathrm{~cm}$; their mean weight, $51.4 \pm 5.1$ $\mathrm{kg}$; their mean muscle mass which was measured by a bioimpedance analysis, $20.5 \pm 2.0 \mathrm{~kg}$; and their mean body mass index, $19.6 \pm 1.5 \mathrm{~kg} / \mathrm{m}^{2}$. All the subjects identified their right legs as their dominant legs.

\section{Methods}

The subjects participated in this study twice, with a one-week interval in between. Three trained male raters of the isokinetic dynamometer (Biodex System 3; Biodex Medical Systems, New York, NY, USA) and HHD (JTECH Medical, Salt Lake City, UT, USA) tests measured the respective peak isometric torque (unit, $\mathrm{Nm}$ ) and the peak muscle force (unit, N) of both of the subjects' knee extensors. On the first day, one rater conducted the isokinetic dynamometer test thrice; and after the HHD was fixed, each of the three raters made measurements three times. On the second day, the HHD was fixed and one of the three raters made three measurements, and then each of the three raters made three measurements using the non-fixed HDD (Fig. 1). The isokinetic dynamometer test and the HHD test were randomly conducted. Each measurement was conducted with a five-minute interval in order to prevent muscle fatigue [17]. All the tests were conducted 1) in the sitting and supine positions and 2) on the right and left legs.

\section{Isokinetic dynamometer test}

For the isokinetic dynamometer test, the subject was seated on a dynamometer chair, and the lateral femoral condyle of her knee joint was aligned with the rotating axis of the dynamometer. Then, her knee joint was flexed from the maximum extension angle by $35^{\circ}$ before her ankle was fixed with a Velcro strip on the rotating axis of the isokinetic dynamometer in order to measure the isometric peak torque $(\mathrm{Nm})$ of the knee extensor (Fig. 2). The flexion position was chosen after considering the study results of high-level surface EMG activity with the knee joint that was flexed by $35^{\circ}[18]$ and the favorable reliability of the test results with the stable subject's knee joint that was flexed by $35^{\circ}$ in the HHD test for the knee extensor torque $[19,20]$.

\section{HHD test}

Similar to the isokinetic dynamometer method, the

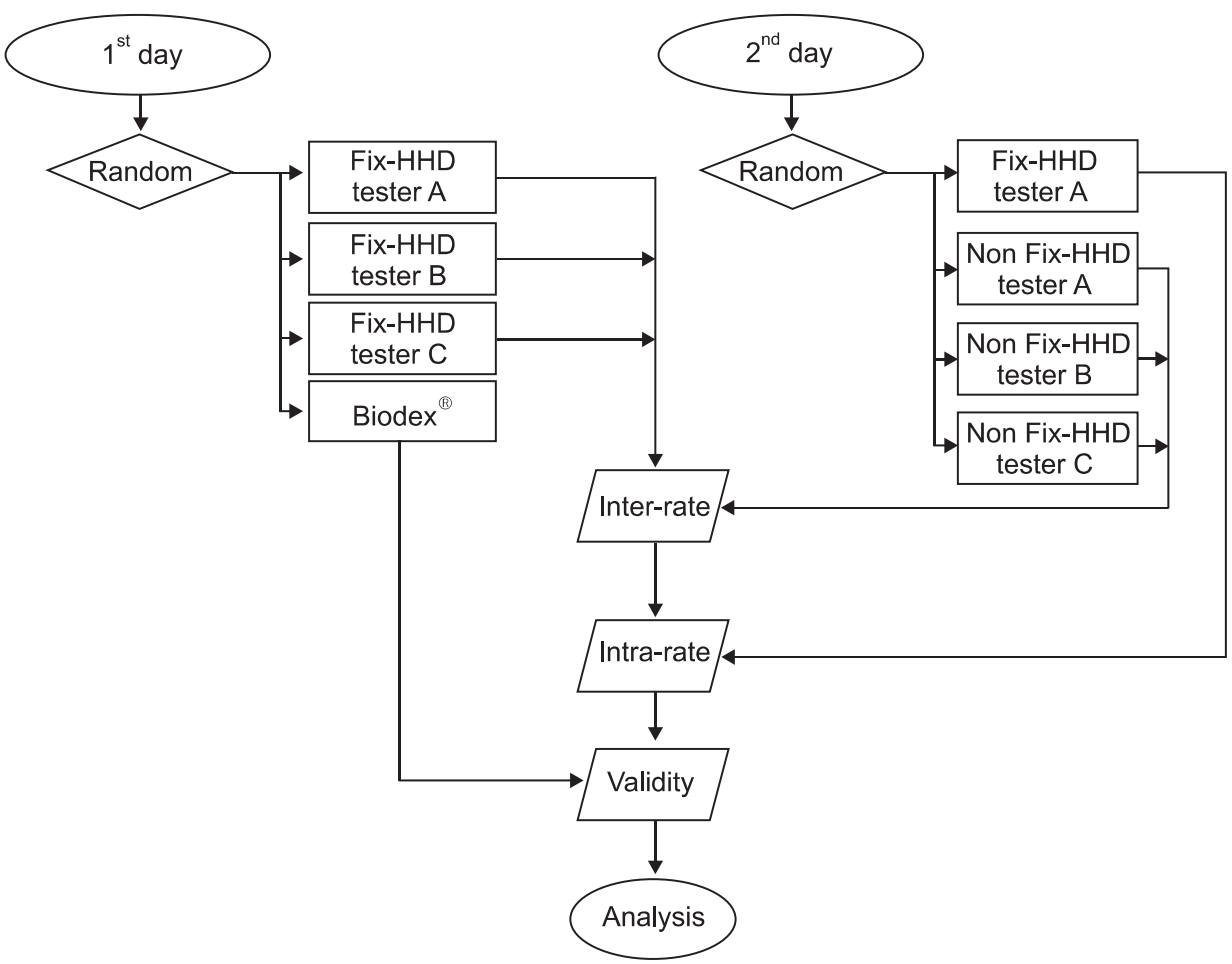

Fig. 1. Flow chart represents a process of the study. 

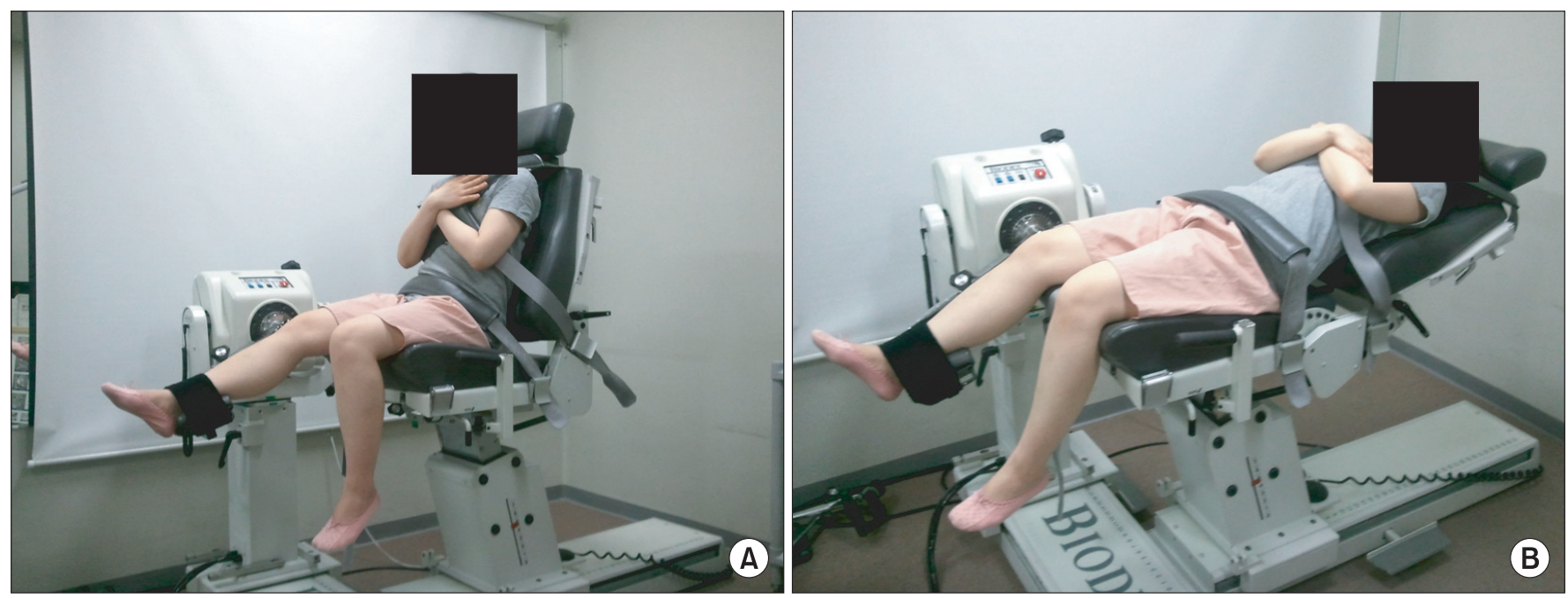

Fig. 2. Study participant positioning for isokinetic dynamometer. (A) Sitting position and (B) supine position.

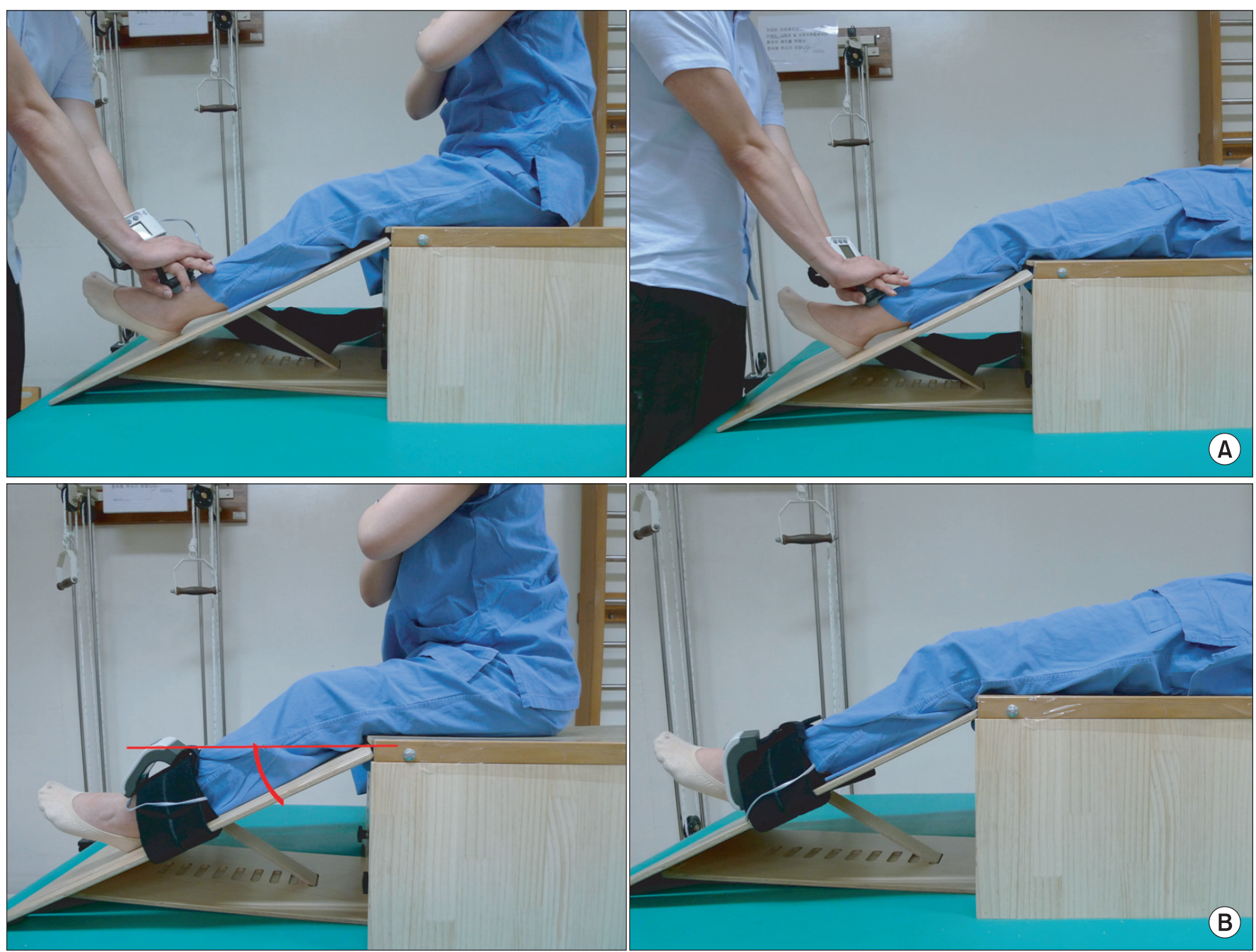

Fig. 3. Study participant positioning for fixed and non-fixed HHD methods. (A) Fixed HHD method with sitting and supine position was observed. (B) Non-fixed HHD method with sitting and supine position was observed. 
subject was in a sitting or supine position, with her knee joint flexed from the maximum extension angle by $35^{\circ}$, after which the HDD was positioned in the area that was fixed with the ankle belt in the isokinetic dynamometer test in order to measure the isometric peak flexion force (N) of both knee joints (Fig. 3). Similar to the isokinetic dynamometer test, the HDD was fixed in the area that was 2 finger breadths above the ankle joint and distal to the tibia using a belt or the rater's hand. When the fixed HHD was used, it was fixed at the lower edge of the slanting surface of the slanting box using a belt. The slanting surface was firmly fixed on the bottom of the box during the test to prevent any movement or lifting (Fig. 3). As the same HDD was used in both the fixed and non-fixed methods, errors that could have occurred due to the discrepancy in the type of HHD were prevented

In the HHD test, the intra-rater reliability was assessed by comparing the results of the first- and second-day tests, which were conducted by the same rater. To assess the inter-rater reliability in the fixed HHD method, each of the three raters measured the peak knee extensor force

Table 1. Descriptive statistics of fixed and non-fixed HHD isokinetic dynamometer (unit, $\mathrm{Nm}$ )

\begin{tabular}{|c|c|c|}
\hline & Mean & SD \\
\hline \multicolumn{3}{|l|}{ Fixed HHD } \\
\hline Supine Rt. ${ }^{\text {a) }}$ & 66.03 & 24.17 \\
\hline Seat Rt. ${ }^{a}$ & 59.22 & 17.44 \\
\hline Supine Lt. & 65.69 & 20.44 \\
\hline Seat Lt. & 58.12 & 17.09 \\
\hline \multicolumn{3}{|c|}{ Non-fixed HHD } \\
\hline Supine $\mathrm{Rt}^{\mathrm{a})}$ & $49.82^{\mathrm{b}, \mathrm{c}} \mathrm{x}$ & 14.05 \\
\hline Seat Rt. ${ }^{\text {a) }}$ & $46.76^{\mathrm{b}, \mathrm{c})}$ & 9.80 \\
\hline Supine Lt. $^{\text {a) }}$ & $56.35^{\mathrm{b}, \mathrm{c})}$ & 15.74 \\
\hline Seat Lt. ${ }^{\text {a) }}$ & 52.66 & 12.85 \\
\hline \multicolumn{3}{|c|}{ Isokinetic dynamometer } \\
\hline Supine Rt. ${ }^{\text {a) }}$ & 69.63 & 18.11 \\
\hline Seat Rt. ${ }^{a}$ & 65.58 & 15.63 \\
\hline Supine Lt. & 66.47 & 19.25 \\
\hline Seat Lt. & 59.67 & 15.60 \\
\hline
\end{tabular}

HHD, hand-held dynamometer; Rt., right; Lt., left; SD, standard deviation.

${ }^{\text {a) }} \mathrm{p}<0.05$ difference between the result of supine position and seat position. ${ }^{b} \mathrm{p}<0.05$ difference between the result of isokinetic dynamometer and non-fixed HHD. ${ }^{c)} p<0.05$ difference between the result of fixed HHD and nonfixed HHD. of both legs three times on the first day. To assess the inter-rater reliability in the non-fixed HHD method, each of the three raters measured the peak knee extensor force of both legs three times on the second day.

\section{Statistical analysis}

To compare all the muscle forces $(\mathrm{N})$ that were measured using the HHD with the peak torque $(\mathrm{Nm})$, which was measured with the isokinetic dynamometer, the length $(\mathrm{m})$ between the knee joint and the ankle was measured and then multiplied by the muscle force $(\mathrm{N})$, which was measured using the HHD, in order to calculate the peak torque $(\mathrm{Nm})$.

To confirm the validity of the peak knee joint torque values that were measured using the fixed and non-fixed HHD tests, the values that were measured in the isokinetic dynamometer test were compared with the results of the paired samples t-test and Pearson correlation analysis.

To assess the reliability of the fixed HHD test, the intraclass correlation coefficient (ICC) and the standard error of measurement were calculated using the test-retest method. To confirm and compare the reliability of the fixed HHD test with the non-fixed HHD test, the interrater ICC and the standard error were calculated and analyzed.

To confirm the difference in the peak knee extensor torque values that were obtained from the fixed HHD, non-fixed HHD, and isokinetic dynamometer tests in the sitting and supine positions, as well as to analyze the

Table 2. Pearson correlation coefficient ( $\mathrm{r}$ ) between fixed HHD and non-fixed HHD methods according to isokinetic dynamometer

\begin{tabular}{|c|c|}
\hline & Pearson correlation coefficient \\
\hline \multicolumn{2}{|l|}{ Fixed HHD } \\
\hline Supine Rt. & $0.806^{*}$ \\
\hline Supine Lt. & $0.524^{*}$ \\
\hline Seat Rt. & $0.473^{*}$ \\
\hline Seat Lt. & 0.294 \\
\hline \multicolumn{2}{|c|}{ Non-fixed HHD } \\
\hline Supine Rt. & 0.185 \\
\hline Supine Lt. & $0.384^{*}$ \\
\hline Seat Rt. & 0.001 \\
\hline Seat Lt. & 0.016 \\
\hline
\end{tabular}

HHD, hand-held dynamometer; Rt., right; Lt., left. ${ }^{*} \mathrm{p}<0.05$ was considered significant. 
validity of the tests, a paired samples t-test and a Pearson correlation analysis were conducted. For statistical analysis, SPSS ver. 17.0 (SPSS Inc., Chicago, IL, USA) was used.

\section{RESULTS}

The mean peak torque values that were obtained from the HHD method in the sitting and supine positions were less than those from the isokinetic dynamometer
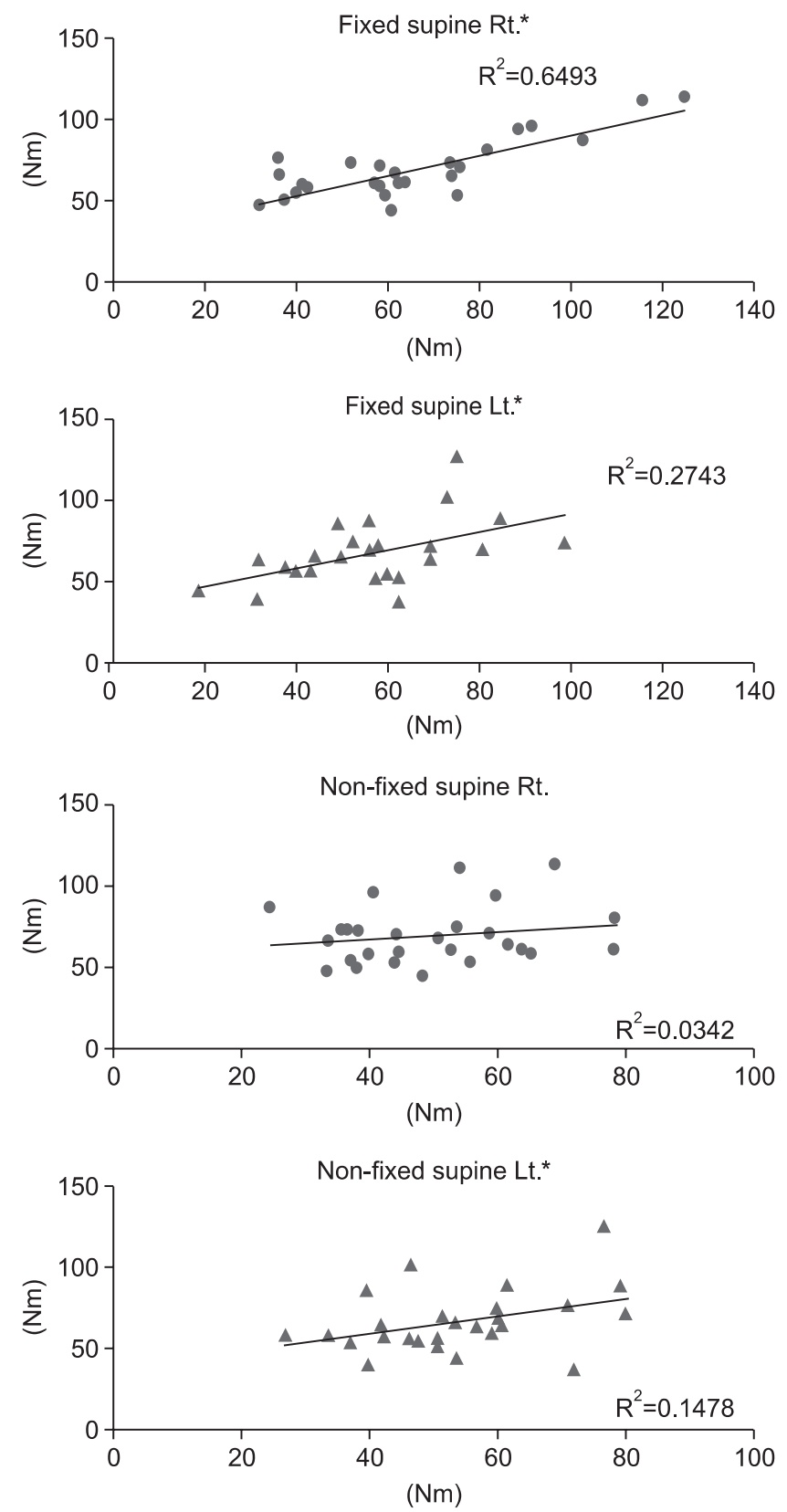

method. However, in the paired samples t-test, no statistically significant difference between the fixed HHD method and the isokinetic dynamometer method was observed. However, the mean peak torque values of the knee extensor, which were obtained from the non-fixed HHD method, showed a statistically significant difference from those obtained from the isokinetic dynamometer method, excluding the left leg value that was measured in the sitting position (Table 1). In the Pearson correlation
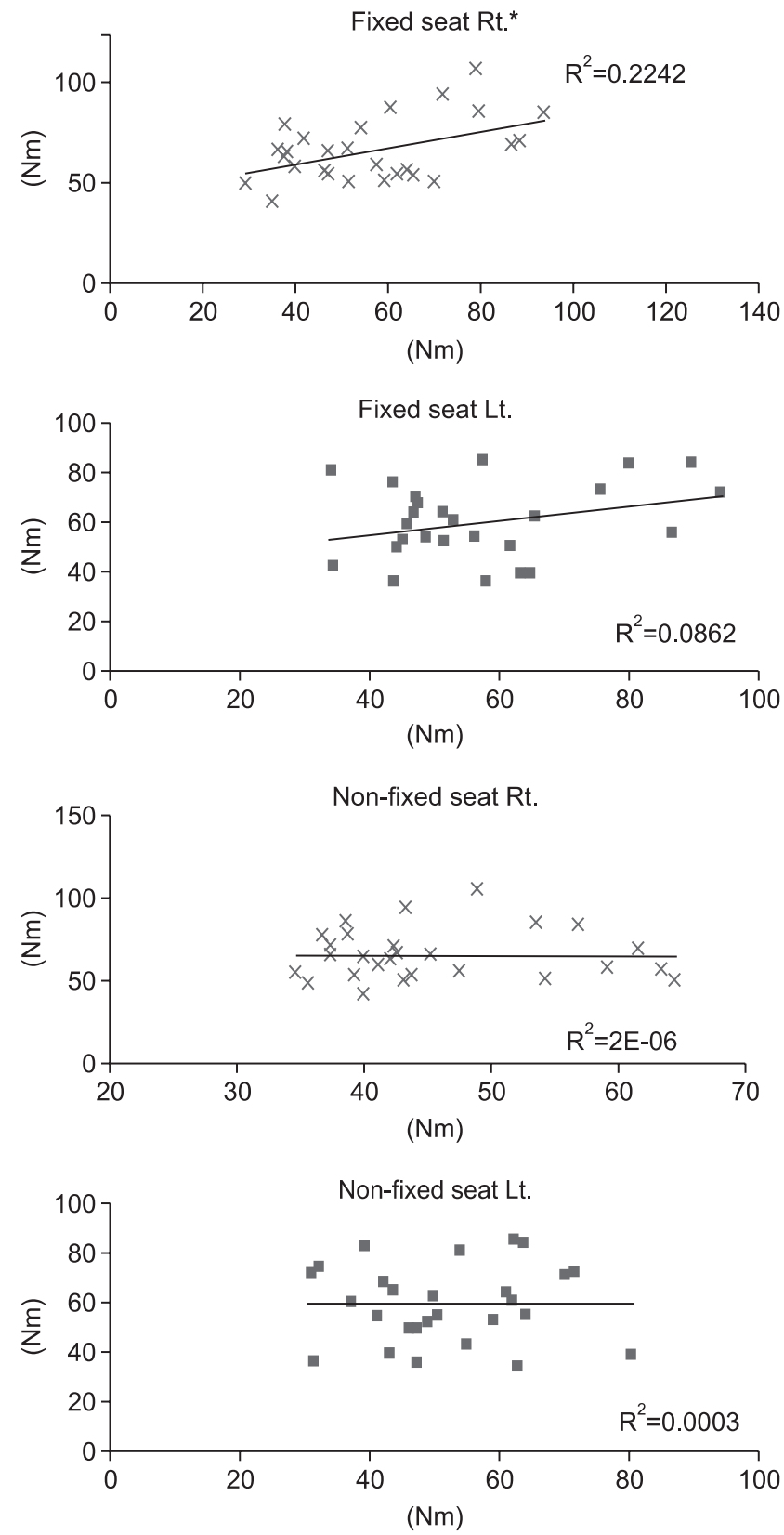

Fig. 4. Correlation of fixed HHD and non-fixed HHD methods (x-axis) with isokinetic dynamometer (y-axis) in a scatter diagram. HHD, hand-held dynamometer; Rt., right; Lt., left. *p $<0.05$ was considered significant. 
Table 3. ICC and SEM of fixed HHD and non-fixed HHD methods by three testers

\begin{tabular}{|lll}
\hline & ICC & SEM \\
\hline Fixed HHD & & \\
\hline Supine Rt. & 0.984 & 2.9 \\
\hline Supine Lt. & 0.952 & 3.88 \\
\hline Seat Rt. & 0.984 & 2.16 \\
\hline Seat Lt. & 0.983 & 2.09 \\
\hline Non-fixed HHD & & \\
\hline Supine Rt. & 0.963 & 2.58 \\
\hline Supine Lt. & 0.95 & 3.39 \\
\hline Seat Rt. & 0.94 & 2.28 \\
\hline Seat Lt. & 0.962 & 2.61 \\
\hline
\end{tabular}

HHD, hand-held dynamometer; ICC, intraclass correlation coefficient; SEM, standard error of measurement; Rt., right; Lt., left.

analysis of the isokinetic dynamometer test results (Table 2 ), the peak torque values (right $r=0.806$ and left $r=0.524$ ) of both knee extensors, which were measured using the fixed HHD method in the supine position, showed a high correlation with those from the isokinetic dynamometer method $(\mathrm{p}<0.05)$. However, in the sitting position, only the right leg value $(r=0.473)$ showed a significant correlation $(\mathrm{p}<0.05)$. In the non-fixed HHD method, only the left knee joint value in the supine position showed a significant Pearson correlation coefficient value $(r=0.384$, $\mathrm{p}<0.05)$, whereas the rest did not show significant values (Fig. 4).

The inter-rater ICC values in the fixed HHD method ranged from 0.952 to 0.984 , and those in the non-fixed HHD method were from 0.940 to 0.963 . Thus, both methods demonstrated significantly high reliability; yet, the fixed HHD method values were higher than the non-fixed HHD method values (Table 3). The intra-rater ICC values in the fixed HHD method ranged from 0.968 to 0.985 , indicating a high reliability (Table 4).

The mean isometric peak torque values of both knee extensors in the supine position were higher than those in the sitting position in both the HHD and isokinetic dynamometer tests (Table 1). The paired samples t-test analysis showed that the peak torque of the right knee extensor values of both the isokinetic dynamometer and fixed HHD tests in the supine position were significantly greater than those in the sitting position, whereas the peak torque of the left knee extensor did not show a sta-
Table 4. Intra-rater reliability of fixed HHD method

\begin{tabular}{ccc}
\hline & ICC & SEM \\
\hline Fixed HHD & & \\
\hline Supine Rt. & 0.976 & 2.48 \\
\hline Supine Lt. & 0.981 & 2.20 \\
\hline Seat Rt. & 0.968 & 2.86 \\
\hline Seat Lt. & 0.985 & 1.96 \\
\hline
\end{tabular}

HHD, hand-held dynamometer; ICC, intraclass correlation coefficient; SEM, standard error of measurement; Rt., right; Lt., left.

tistically significant difference. In the non-fixed HHD test, the values of the peak torque of both knee extensors in the supine position were significantly greater than those in the sitting position (Table 1). As a result of the Pearson correlation analysis, the correlation coefficients of the fixed HHD measurements obtained in the supine position were 0.806 (right) and 0.524 (left), which show a high correlation with those of the isokinetic dynamometer measurements $(\mathrm{p}<0.05)$. However, in the sitting position, only the right leg values showed such a correlation, with a 0.473 correlation coefficient $(\mathrm{p}<0.05)($ Table 2$)$. In the Pearson correlation analysis, only the non-fixed HHD measurements in the supine position from the left leg showed a correlation with the isokinetic dynamometer measurements, with a 0.384 correlation coefficient $(\mathrm{p}<0.05)$ (Table 2).

\section{DISCUSSION}

HHD is inexpensive, easy to carry and use. However, many factors affect the test results, and its reliability is known to not be very high. In this study, a test method with the advantage of the HHD and of enhanced reliability is suggested and compared with the isokinetic dynamometer method in order to confirm its validity. When the HHD was fixed on the leg using a specially prepared band, higher validity and reliability were observed than when the rater held it with one hand. In terms of the position, higher validity was observed in the supine position than in the sitting position.

Other than the HHD fixation method, which was an independent variable and the test position, factors that could have affected the reliability of the HHD test results were attempted to be controlled in this study. In the HHD test, the make-test is the method with which the 
subject applied a force to the HHD while the rater was trying not to move it. The break-test is the method with which the rater attempted to overcome the force that the subject applied to his/her ankle using the HHD. The reliability of the make-test is known to be higher than that of the break-test [21]; thus, the non-fixed or handheld method was measured using the make-test method in this study. The reliability of the HHD test is known to increase when the rater is stronger than the subject [22]. In this study, male raters who had strong muscle forces and were trained with the HDD test were selected to control their muscle force and proficiency. Through the HHD fixation, the factors which could affect the rater or the subject in the non-fixed method were excluded. When the knee extensor strength values in the fixed and non-fixed HHD tests were compared with those in the isokinetic dynamometer test, no statistically significant difference was observed between the fixed HHD and isokinetic dynamometer; however, most of the non-fixed HHD test values were confirmed to have significantly differed from those of the isokinetic dynamometer test. Unlike the isokinetic dynamometer and fixed HHD tests, the non-fixed HHD test conveyed a high peak torque value in the left leg. The reliability of the test-retest method was high regardless of the fixation type, but that of the fixed method was confirmed to have been higher than that of the non-fixed method. The difference that was observed between the fixed and non-fixed methods might have been due to systemic error [23]. A systemic error is a bias that consistently occurs in a specific direction, revealing a correspondence between the measured value and the true value. When the rater resists the subject's leg force to maintain his/her measurement position, a systemic error increases in case the rater's force against the subject is not strong enough. After the HHD was fixed on the portable device using a belt, the systemic error decreased and the validity was enhanced, unlike in the non-fixed HHD method.

In the fixed HHD test with the $35^{\circ}$ flexed knee joint, the values in the supine position and of the right leg showed the greatest correlation with those of the isokinetic dynamometer test. With the $90^{\circ}$ knee joint, the isometric extensor muscle force in the sitting position was reportedly $10.6 \%$ greater than in the supine position [18]; however, in the study with the $35^{\circ}$ flexed knee joint, higher measured values (right $5.82 \%$ and left $10.23 \%$ ) were ob- tained in the supine position than in the sitting position. This might have been due to the optimal muscle length at the moment of the peak muscle force [24] and to the muscle length, which changes according to the angles of the knee and hip joints in the sitting or supine positions. According to the previous study, the isometric muscle force of the rectus femoris muscle was confirmed to have been greater with the $35^{\circ}$ flexed knee joint than with the $75^{\circ}$ flexed knee joint, through the measurement of the electromyography signals [25]. In addition, the reliability of the values in the supine position was higher than in the sitting position, because the supine position was more convenient than the sitting position for the elderly subjects [26] and moreover, it was deemed more stable in maintaining the measurement position during the test period. To form a definitive conclusion, further studies on the position may be needed in the future.

The accuracy of the right leg measurement was superior to that of the left leg measurement because the right leg was the dominant leg in all the subjects. However, no standard on the differentiation of the dominant leg from the non-dominant leg could be prepared in this study. Before the reliability of the determination of the dominant leg is evaluated, an accurate method of determining the dominant leg should be established; hence, additional validation studies may be needed for left leg dominance.

One of the limitations of this study is that the subjects were limited to young women. As a preliminary study to investigate the reliability and validity of the HHD test according to the position and type of the fixation, the subjects were restricted to young women in order to control the variables and to simplify the study structure. To generalize the results of this study, additional studies with both men and women, in addition to the elderly who clinically need measurement of their leg muscle strength, may be needed. The other limitation of this study is the random allocation of measurement. On the first day, the isokinetic dynamometer test and the fixed HHD test were randomly conducted; and on the second day, the fixed HHD test and the non-fixed HHD test were performed. If the fixed HHD, non-fixed HHD, and isokinetic dynamometer tests had been randomly conducted, the results of this study might have been more reliable. However, to investigate the inter-rater reliability of the test-retest fixed HHD method, the fixed HHD test was conducted over 
two days, and the overall quantity of exertion among the subjects and the raters was evenly distributed, taking into consideration their muscle fatigue and concentration deterioration. Nevertheless, considering the high intrarater reliability (ICC, $0.968-0.985$ ) of the test-retest result of the fixed HHD method and the high Pearson correlation results of the fixed HHD test, which was conducted on the second day (results not shown), along with the isokinetic dynamometer test, the reliability was deemed to have not been significantly affected.

In measuring the knee extensor strength with an HHD, the fixation of HHD during measurement in the supine position was confirmed to increase the reliability and validity in measuring quadriceps strength in this study. Because the fixed HHD method is not only more economical than the isokinetic dynamometer method, but is also easy to carry and use, it may be able to use in place of the isokinetic dynamometer method in clinical or research settings.

\section{CONFLICT OF INTEREST}

No potential conflict of interest relevant to this article was reported.

\section{REFERENCES}

1. Bean JF, Kiely DK, Herman S, Leveille SG, Mizer K, Frontera WR, et al. The relationship between leg power and physical performance in mobility-limited older people. J Am Geriatr Soc 2002;50:461-7.

2. Suzuki T, Bean JF, Fielding RA. Muscle power of the ankle flexors predicts functional performance in community-dwelling older women. J Am Geriatr Soc 2001;49:1161-7.

3. Lauretani F, Russo CR, Bandinelli S, Bartali B, Cavazzini C, Di Iorio A, et al. Age-associated changes in skeletal muscles and their effect on mobility: an operational diagnosis of sarcopenia. J Appl Physiol 2003;95:1851-60.

4. Al Snih S, Markides KS, Ottenbacher KJ, Raji MA. Hand grip strength and incident ADL disability in elderly Mexican Americans over a seven-year period. Aging Clin Exp Res 2004;16:481-6.

5. Brinker MR, Cuomo JS, Popham GJ, O'Connor DP, Barrack RL. An examination of bias in shoulder scor- ing instruments among healthy collegiate and recreational athletes. J Shoulder Elbow Surg 2002;11:463-9.

6. Incel NA, Ceceli E, Durukan PB, Erdem HR, Yorgancioglu ZR. Grip strength: effect of hand dominance. Singapore Med J 2002;43:234-7.

7. Knapik JJ, Wright JE, Mawdsley RH, Braun JM. Isokinetic, isometric and isotonic strength relationships. Arch Phys Med Rehabil 1983;64:77-80.

8. Farrell M, Richards JG. Analysis of the reliability and validity of the kinetic communicator exercise device. Med Sci Sports Exerc 1986;18:44-9.

9. Drouin JM, Valovich-mcLeod TC, Shultz SJ, Gansneder BM, Perrin DH. Reliability and validity of the Biodex System 3 Pro isokinetic dynamometer velocity, torque and position measurements. Eur J Appl Physiol 2004;91:22-9.

10. Feiring DC, Ellenbecker TS, Derscheid GL. Test-retest reliability of the Biodex isokinetic dynamometer. J Orthop Sports Phys Ther 1990;11:298-300.

11. Toonstra J, Mattacola CG. Test-retest reliability and validity of isometric knee flexion and extension measurement using three methods of assessing muscle strength. J Sport Rehabil 2012 Sep 4 [Epub]. http:// journals.humankinetics.com/jsr-technical-reports/ jsr-technical-reports/testndashretest-reliability-andvalidity-of-isometric-knee-flexion-and--extensionmeasurement-using-3-methods-of-assessing-muscle-strength.

12. Scott DA, Bond EQ, Sisto SA, Nadler SF. The intraand interrater reliability of hip muscle strength assessments using a handheld versus a portable dynamometer anchoring station. Arch Phys Med Rehabil 2004;85:598-603.

13. Kollock RO Jr, Onate JA, Van Lunen B. The reliability of portable fixed dynamometry during hip and knee strength assessments. J Athl Train 2010;45:349-56.

14. Keating JL, Matyas TA. The influence of subject and test design on dynamometric measurements of extremity muscles. Phys Ther 1996;76:866-89.

15. Stark T, Walker B, Phillips JK, Fejer R, Beck R. Handheld dynamometry correlation with the gold standard isokinetic dynamometry: a systematic review. PM R 2011;3:472-9.

16. Thorborg K, Bandholm T, Holmich P. Hip- and kneestrength assessments using a hand-held dynamometer with external belt-fixation are inter-tester reliable. 
Knee Surg Sports Traumatol Arthrosc 2013;21:550-5.

17. Woods S, Bridge T, Nelson D, Risse K, Pincivero DM. The effects of rest interval length on ratings of perceived exertion during dynamic knee extension exercise. J Strength Cond Res 2004;18:540-5.

18. Maffiuletti NA, Lepers R. Quadriceps femoris torque and EMG activity in seated versus supine position. Med Sci Sports Exerc 2003;35:1511-6.

19. Walsworth M, Schneider R, Schultz J, Dahl C, Allison S, Underwood F, et al. Prediction of 10 repetition maximum for short-arc quadriceps exercise from handheld dynamometer and anthropometric measurements. J Orthop Sports Phys Ther 1998;28:97-104.

20. Knapik JJ, Wright JE, Mawdsley RH, Braun J. Isometric, isotonic, and isokinetic torque variations in four muscle groups through a range of joint motion. Phys Ther 1983;63:938-47.

21. Stratford PW, Balsor BE. A comparison of make and break tests using a hand-held dynamometer and the Kin-Com. J Orthop Sports Phys Ther 1994;19:28-32.
22. Deones VL, Wiley SC, Worrell T. Assessment of quadriceps muscle performance by a hand-held dynamometer and an isokinetic dynamometer. J Orthop Sports Phys Ther 1994;20:296-301.

23. Taylor JR. An introduction to error analysis: the study of uncertainties in physical measurements. 2nd ed. Sausalito, CA: University Science Books; 1999.

24. Visser JJ, Hoogkamer JE, Bobbert MF, Huijing PA. Length and moment arm of human leg muscles as a function of knee and hip-joint angles. Eur J Appl Physiol Occup Physiol 1990;61:453-60.

25. Babault N, Pousson M, Michaut A, Van Hoecke J. Effect of quadriceps femoris muscle length on neural activation during isometric and concentric contractions. J Appl Physiol 2003;94:983-90.

26. Martin HJ, Yule V, Syddall HE, Dennison EM, Cooper C, Aihie Sayer A. Is hand-held dynamometry useful for the measurement of quadriceps strength in older people? A comparison with the gold standard Biodex dynamometry. Gerontology 2006;52:154-9. 